\title{
Far-from-Equilibrium Route to Superthermal Light in Bimodal Nanolasers
}

\author{
Mathias Marconi, ${ }^{1}$ Julien Javaloyes, ${ }^{2}$ Philippe Hamel, ${ }^{1, \dagger}$ Fabrice Raineri, ${ }^{1,3}$ Ariel Levenson, ${ }^{1}$ and Alejandro M. Yacomotti ${ }^{1, *}$ \\ ${ }^{1}$ Centre de Nanosciences et de Nanotechnologies, CNRS, Université Paris-Sud, \\ Université Paris-Saclay, C2N-Marcoussis, 91460 Marcoussis, France \\ ${ }^{2}$ Departament de Física, Universitat de les illes Balears, C/ Valdemossa km 7.5, 07122 Mallorca, Spain \\ ${ }^{3}$ Université Paris diderot, Sorbonne Paris Cité, 73013 Paris, France
}

(Received 11 August 2017; published 31 January 2018)

\begin{abstract}
Microscale and nanoscale lasers inherently exhibit rich photon statistics due to complex light-matter interaction in a strong spontaneous emission noise background. It is well known that they may display superthermal fluctuations - photon superbunching - in specific situations due to either gain competition, leading to mode-switching instabilities, or carrier-carrier coupling in superradiant microcavities. Here we show a generic route to superbunching in bimodal nanolasers by preparing the system far from equilibrium through a parameter quench. We demonstrate, both theoretically and experimentally, that transient dynamics after a short-pump-pulse-induced quench leads to heavy-tailed superthermal statistics when projected onto the weak mode. We implement a simple experimental technique to access the probability density functions that further enables quantifying the distance from thermal equilibrium via the thermodynamic entropy. The universality of this mechanism relies on the far-from-equilibrium dynamical scenario, which can be mapped to a fast cooling process of a suspension of Brownian particles in a liquid. Our results open up new avenues to mold photon statistics in multimode optical systems and may constitute a test bed to investigate out-of-equilibrium thermodynamics using micro or nanocavity arrays.
\end{abstract}

DOI: 10.1103/PhysRevX.8.011013

Subject Areas: Photonics Statistical Physics

\section{INTRODUCTION}

The ability to tailor specific correlation properties of light is of central importance in quantum optics and also of practical interest in applications such as the generation of multiphoton effects [1] and time-domain ghost imaging [2]. Light-emitting semiconductors combined with engineered photonic environments-e.g., optical cavities-prove useful in this context as they enable great control over the photon statistics from incoherent [spontaneous emission (SE)] to coherent (laser) sources [3].

An ideal incoherent light source is a blackbody, where the thermal equilibrium of a photon gas produces chaotic fluctuations, accurately described by exponential distributions of the photon number $n$,

$$
P(n)=\frac{\langle n\rangle^{n}}{(1+\langle n\rangle)^{1+n}} \approx \frac{1}{\langle n\rangle} e^{-n /\langle n\rangle}
$$

\footnotetext{
*Alejandro.Giacomotti@u-psud.fr

${ }^{\dagger}$ Present address: Cabinet Camus-Lebkiri, Immeuble Stratege A, 51 Rue Ampere, 31670 Toulouse, France.

Published by the American Physical Society under the terms of the Creative Commons Attribution 4.0 International license. Further distribution of this work must maintain attribution to the author(s) and the published article's title, journal citation, and DOI.
}

where the approximation holds in the large $n$ limit. Photon distributions as in Eq. (1) can be generated by, e.g., SE in semiconductor media as a result of radiative carrier recombinations in bulk or spatially confined direct bandgap materials. The degree of second order coherence at zero time delay, $g^{(2)}(\tau=0)$, is useful to quantify the thermal fluctuations: $g^{(2)}(0)=2$ is the thermal limit corresponding to chaotic light [Eq. (1)], whereas $g^{(2)}(0)>2$ stands for superthermal light. On the other hand, laser radiation leads to coherent emission properties well described by Poissonian distributions $\left[g^{(2)}(0)=1\right]$.

Superthermal light statistics has already been observed in a number of photonic systems such as rotating diffusive plates [4], superradiant quantum dots [5], or micropillars showing switching instabilities between two orthogonally polarized modes [6,7]. Superthermal fluctuations have also been reported in the spatial domain as a result of light propagation in disordered waveguide arrays $[8,9]$. In all these cases, (quantum or classical) light and/or matter coupling mechanisms at (quasi)equilibrium are the main ingredients to generate extra correlations.

In this article, we show a dynamical route toward superbunching relying on far-from-equilibrium states following a rapid variation of a parameter, or "quench" (see, e.g., Refs. [10-12]), in semiconductor-coupled nanolasers. This takes place in the strong intercavity coupling limit or "Rabi regime" in the terminology of Bose-Einstein condensates 
$[13,14]$, where the intersite coupling overcomes the nonlinear interaction energy. Specifically, we show that such nonequilibrium states for the transient mode energy flow after a quench naturally produce superthermal, long-tailed statistical distributions, of a different nature than typical bimodal distributions previously observed in microlasers. Furthermore, the dynamical equations can be mapped onto a 1D Langevin equation governing the fast cooling of an ensemble of Brownian particles under gravity. The limit of validity of this $1 \mathrm{D}$ stochastic model is subsequently tested by accessing the Josephson regime - cavity coupling lower than the nonlinearity - which may lead to self-trapping [15-17], multistability [18], spontaneous symmetry breaking and phase transitions [19-22], and even quantum criticality $[23,24]$. Thus, our quenched bimodal nanolaser belongs to a large class of far-from-equilibrium systems where superthermal statistics is the consequence of transient transport mechanisms in phase space dominating over noise diffusion.

This paper is organized as follows. In Sec. II we introduce the far-from-equilibrium dynamical theory following short pump pulses in our coupled cavity system. Section III presents the experimental setup and the technique to measure the full probability distributions of the output pulse energies. It shows the experimental results on long-tailed superthermal light, which are subsequently discussed and compared with the theoretical predictions. Section IV is devoted to the analogy with the fast cooling of Brownian particles. Conclusions and perspectives are given in Sec. V.

\section{CLASSICAL OPTICAL QUENCH THEORY}

In this section we will show, using a stochastic semiclassical model for evanescently coupled cavities, that transients after quench can be approximated by "diffusion-free" nonequilibrium solutions of a 1D Langevin equation for the fractional mode population imbalance $x=\left(I_{B}-I_{A B}\right) /$ $\left(I_{B}+I_{A B}\right)$, where $I_{B(A B)}$ are the intensities of the bonding (antibonding) hybrid modes.

We model the dynamics of two coupled semiconductor nanolasers using coupled-mode equations (see Supplemental Material [25], Sec. A). The dynamical variables are the normalized slowly varying cavity fields $A_{1,2}$ and normalized carrier population inversions $D_{1,2}$, where the indexes $j=1$ and $j=2$ stand for left and right cavities, respectively. The coupling between the two cavities is governed by the dimensional parameters $\gamma$ and $K$ that represent the loss and frequency difference between the two eigenmodes normalized to the cavity photon rate $\kappa\left(A_{1}=A_{2}\right.$, bonding, and $A_{1}=-A_{2}$, antibonding). We assume strong intercavity coupling $K \gg 1$; i.e., we operate in the Rabi quasilinear oscillating regime. We furthermore define $A_{j}=$ $\sqrt{2 I_{j}} \exp \left(i \psi_{j}\right)$ and $D_{1,2}=N \pm n$, where $N$ and $n$ represent the population average and the difference between the two carrier reservoirs, respectively. In the strong coupling limit, the original deterministic equations can be written as two subsets of equations. The first one is a $2 \mathrm{D}$ dynamical system coupling the total intensity $I=I_{1}+I_{2}$ and the average carrier density $N$, which, at leading order of both $\gamma \ll 1$ and $n \ll 1$, reads

$$
\dot{I}=2(N-1) I, \quad \gamma_{\text {tot }}^{-1} \dot{N}=P(t)-N(1+I) .
$$

The subsystem in Eqs. (2) parametrically forces a second subset of equations, the Bloch sphere, through the variable $n$ (see Supplemental Material [25], Sec. B),

$$
\begin{aligned}
\dot{\theta} & =-n \sin 2 \theta+\gamma \cos 2 \theta \cos \Phi-K \sin \Phi \\
\dot{\Phi} & =2 \alpha n-2 \gamma \frac{\sin \Phi}{\sin 2 \theta}-2 K \frac{\cos \Phi}{\tan 2 \theta} \\
\gamma_{\text {tot }}^{-1} \dot{n} & =-n(1+I)-N I \cos 2 \theta,
\end{aligned}
$$

where $\gamma_{\text {tot }} \ll 1$ is the ratio of the total carrier recombination to the cavity rates, $\theta=2 \arctan \left(\sqrt{I_{2} / I_{1}}\right) \in[0, \pi]$ is the polar angle, which is a measure of the photon density imbalance between the two cavities, and the azimuthal angle $\Phi=\psi_{1}-\psi_{2}$ is the phase difference between the sites. In this representation, the bonding and antibonding modes correspond to two opposite points over the equator, $(\theta, \Phi)=$ $(\pi / 2,0)$ and $(\theta, \Phi)=(\pi / 2, \pi)$ (see Fig. 1).

We deal with a transient, far-from-equilibrium situation, as a consequence of a short incoherent pump pulse $P(t)$. A particular solution takes the form of a trajectory in phase space that eventually comes back to the neighborhood of the rest equilibrium point $\left(\left|A_{j}\right|^{2}=0\right)$. The stochastic fluctuations coming from SE noise, which are inherent to micro or nanolasers [26-28] and are noise source terms in Eqs. (2)-(5), have a main impact: the trajectory in phase space triggered by a given pump pulse will start from random initial phases.

The averaged intensity resulting from Eqs. (2) with SE noise and a short pump pulse is shown in Fig. 1(a) as a function of time. We consider repetitive sequential cycles of optical pumping such that each response to a single pump pulse is a particular statistical realization on the Bloch sphere [Eqs. (3)-(5)], so that we can follow an ensemble of trajectories in parallel $\left(10^{4}\right)$. The results are shown for different elapsed times before [Fig. 1(b), right] and within [Figs. 1(c)-1(d), right] the $I(t)$ laser pulse. We can observe two distinct situations: a quasiequilibrium random phase distribution below threshold [Fig. 1(b), right] and nonequilibrium [Figs. 1(c)-1(d), right], which becomes peaked around the antibonding fixed point $(\theta, \Phi)=(\pi / 2, \pi)$ at the $I(t)$ maximum [Fig. 1(d), right].

The main statistical information of the out-ofequilibrium mode energy exchange is contained in the coordinate $x=\sin \theta \cos \Phi$ of the Bloch sphere, which can be shown to account for the fractional mode population imbalance $x=\left(I_{B}-I_{A B}\right) /\left(I_{B}+I_{A B}\right)$, whereas the coordinate $z=\cos \theta$ is the fractional site population imbalance 

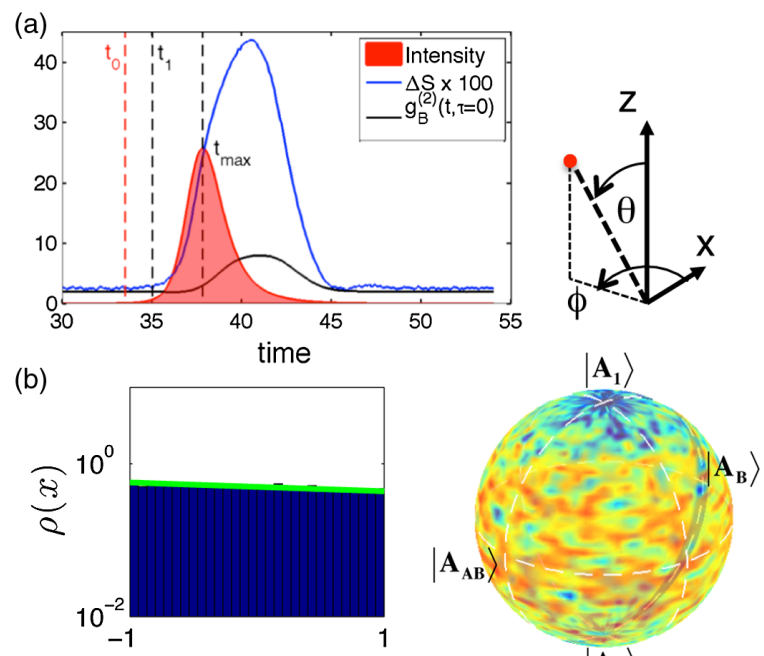

(c)
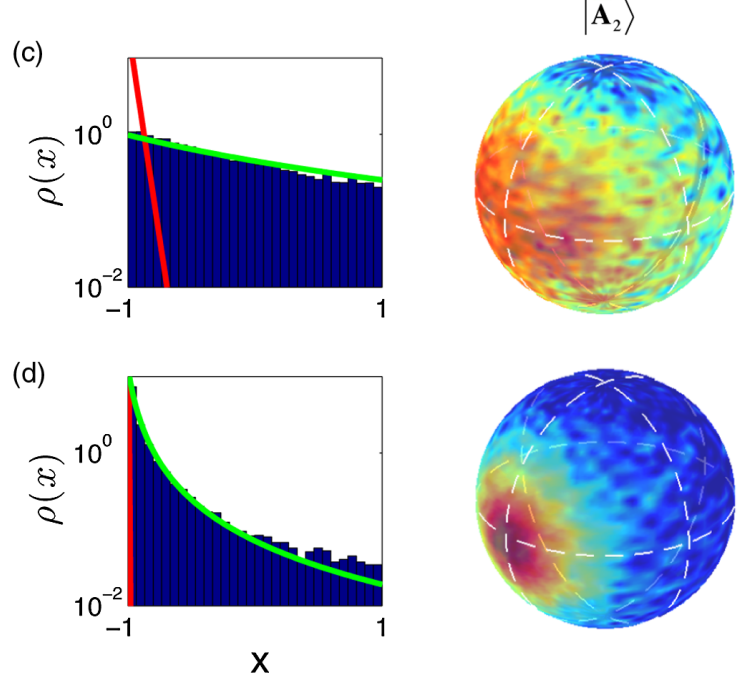

FIG. 1. Numerically simulated response of a coupled nanolaser system under short-pulse pumping (centered at $t_{\text {pump }}=32$ ). (a) Evolution of total laser output intensity (red), excess entropy (blue), and second-order autocorrelation for the bonding mode (black). (b)-(d) Snapshots of the PDFs for the fractional population imbalance (left) and Bloch sphere representations (right) at (b) $t=20$ (before laser buildup), (c) $t_{1}=35$ (during the early phase buildup), and (d) $t_{\max }=37.8$ (pulse maximum). Red curves on PDFs represent the equilibrium solutions of Eq. (7) to a final temperature as given by the instantaneous intensity. Green curves are $T=0$, "free-falling" approximations of the PDF [Eq. (9)] with fitted $t_{0}=33.5$. The simulation parameters are $K=7, \gamma=-0.1, \alpha=5, P=4 P_{\text {th }}$, and $\gamma_{\text {tot }}^{-1}=28$.

$z=\left(I_{1}-I_{2}\right) /\left(I_{1}+I_{2}\right)$. The dynamical evolution of $x$ is governed by the following Langevin equation,

$$
\frac{d x}{d t}=f(x)+g(x) \xi(t)
$$

where $\quad f(x)=\gamma_{x}\left(1-x^{2}\right)-S_{\mathrm{sp}} I^{-1} x \quad$ and $\quad g^{2}(x)=$ $\left(1-x^{2}\right) S_{\mathrm{sp}} I^{-1}$ are the normalized drift and diffusion coefficients, respectively; $\gamma_{x}(t)=2 \gamma-\alpha N I \gamma_{\text {tot }}(2 K)^{-1}$ is the effective damping coefficient $[\alpha$ is the phase-amplitude coupling factor of the semiconductor laser and $S_{\mathrm{sp}}$ the spontaneous emission factor (see Supplemental Material [25], Secs. C-E)]; and $\xi(t)$ is Gaussian noise with zero mean and variance one. Equation (6) can be associated with the Fokker-Planck equation governing the evolution of the probability distribution $\rho(x)$,

$$
\frac{\partial \rho}{\partial t}+\frac{\partial}{\partial x}(f \rho)=\frac{1}{2} \frac{\partial^{2}}{\partial x^{2}}\left(g^{2} \rho\right) .
$$

Although diffusion is given by a multiplicative noise term, the steady-state equilibrium is exponential,

$$
\rho_{\mathrm{eq}}(x ; \beta)=\mathcal{N} e^{-\beta x},
$$

with $\beta=-2 \gamma_{x} I S_{\mathrm{sp}}^{-1}$ and $\mathcal{N}=\beta /(2 \sinh \beta)$, in such a way that $\beta^{-1} \sim S_{\mathrm{sp}} I^{-1}$ can be identified as the effective temperature of a reservoir. Hence, the steep intensity increase [Fig. 1(a)], with buildup time scale corresponding to the cavity photon lifetime, $\dot{I}(t) / I(t) \sim 1$, can be related to a cooling process much faster than the typical time scale of the mode energy exchange $\gamma_{x}^{-1} \sim 10$. This process is analogous to the rapid cooling of an ensemble of Brownian particles in a fluid, as we will show in Sec. IV. The probability distribution is initially at the exponential equilibrium corresponding to the high temperature $\beta=\beta_{0}$ [Fig. 1(b)], for which the statistical distribution of the weak mode is also exponential, as expected from Eq. (1). As $I(t)$ dramatically builds up, the system is forced to evolve in a basically noiseless situation $(\beta \rightarrow \infty)$. In this initial phase of relaxation toward a much lower reservoir temperature, drift dominates over diffusion in Eq. (7) and we can solve it by setting $S_{\mathrm{sp}}=0$. We find that the solution of the transport problem with initial condition $\rho_{t}\left(x, t_{0}\right)=\rho_{0}(x)$ reads

$$
\rho_{t}(x, t)=\frac{4 e^{\tau} \rho_{0}\left[\frac{1+x+e^{\tau}(x-1)}{1+x-e^{\tau}(x-1)}\right]}{\left[1+e^{\tau}+x\left(1-e^{\tau}\right)\right]^{2}},
$$

where we defined the effective normalized time $\tau(t)=$ $\int_{t_{0}}^{t} 2 \gamma_{x}(s) d s$ that takes into account the temporal dependence of $\gamma_{x}(t)$ via the intensity carrier product $N(t) I(t)$. Therefore, $\tau$ may increase faster than $t$ in the time interval around the pulse where $\alpha N I>4 \gamma K \gamma_{\text {tot }}^{-1}$. Equation (9) can thus be regarded as the zero final temperature evolution or "diffusion-free" transient distribution following a short pulse quench, where the only fitting parameter is $t_{0}$ [see Figs. 1(b)-1(d), left, green lines].

We characterize these out-of-equilibrium states by first computing the time-dependent second-order coherence of the bonding mode at vanishing time delay, $g^{(2)}(t, 0)$, plotted in Fig. 1(a). Before and after the laser pulse, i.e., for $I(t) \approx 0, g^{(2)}(t, 0) \approx 2$, which corresponds to thermal emission. Close to the pulse maximum $\left(t_{\max }\right)$, the B mode becomes superthermal, $g^{(2)}\left(t=t_{\max }, 0\right) \approx 3$. After-pulse superbunching can also be observed, $g^{(2)}\left(t=t_{2}, 0\right) \approx 8$. 
Our last step is to use the information of the full statistical distributions [also experimentally accessible (see Sec. III)] to quantify deviations from equilibrium by means of the entropy $S(t)=-\int \rho(x, t) \ln [\rho(x, t)] d x$. To this aim, we consider, starting from $\rho(x, t)$ as the initial state, a subsequent irreversible transformation without net energy exchange with the reservoir. Namely, we define the final state as an equilibrium distribution of temperature $T_{f}$ such that the internal energy is conserved, i.e., $U_{\mathrm{eq}}\left(T_{f}\right)=U(t)$; hence $T_{f}=T_{f}[U(t)]$, where $U(t)=\int x \rho(x, t) d x$ is a timedependent internal energy and the equilibrium distribution is given in Eq. (8). The exchanged entropy with the reservoir thus vanishes, and the only source of entropy production is the degree of internal order; we then define the excess entropy inside the system $\Delta S_{i}(t)$ as

$\Delta S_{i}(t)=S_{\text {eq }}\left\{T_{f}[U(t)]\right\}+\int \rho(x, t) \ln [\rho(x, t)] d x$,

which is zero if the initial state is an equilibrium distribution, and positive otherwise. As such, it quantifies the degree of internal residual order-or coherence-of the system, which is maximum for the farthest nonequilibrium state (see Supplemental Material [25], Secs. G-K, for more details). Figure 1(a) shows $\Delta S_{i}(t)$, which is significantly high close to the pulse maximum, $\Delta S_{i}\left(t_{\max }\right) \approx 0.25$, and can be considered as a measure of the maximum distance from equilibrium than can be measured after quench upon pulse integration.

\section{EXPERIMENTAL REALIZATION}

In order to experimentally show superthermal, longtailed probability density function (PDF) transients after quench in a photonic system, we use two coupled nanolasers operating at room temperature (see Appendix A) under short-pulsed pumping, which is a natural path to experimentally access quench dynamics. Two coupled photonic crystal cavities are optically pumped at the geometrical center with short (100-ps duration) pulses with a period $T_{\text {pump }}=10 \mu \mathrm{s}$ [Fig. 2(a)]. The emission is collected with a high numerical aperture microscope objective, and its back focal plane is imaged through a lens to obtain the far-field pattern [Fourier plane, Fig. 2(a)].

In order to temporally resolve the pulse energy of the two coupled modes $B$ and $A B$, we set up two confocal detection paths: two single-mode fibers are used as pinholes to simultaneously select two small regions on the Fourier plane and detect their intensities. These regions are located at the center $(k=0)$ for the B-mode detection and shifted along the $x$ direction for the AB mode [Fig. 2(a)]. Signals are sent to two identical low-noise $(200 \mathrm{fW} / \sqrt{\mathrm{Hz}}), 660$ MHz-bandwidth avalanche photodiode (APD) detectors. Hence, the detectors integrate the output pulses, resulting in peak APD signals proportional to the pulse energy, which is
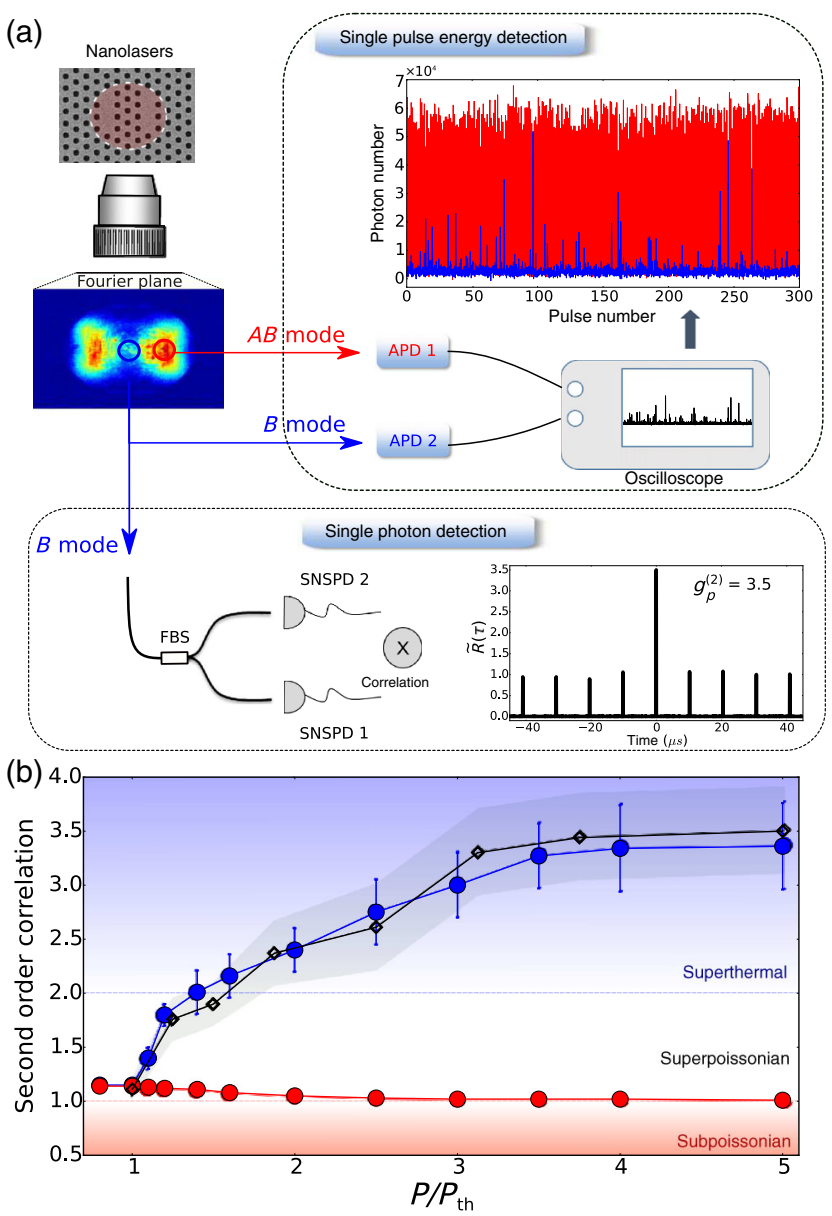

FIG. 2. Experimental autocorrelation results. (a) Top: "Singlepulse energy detection" scheme using the pulse-to-pulse measurement technique of emitted photon numbers. Simultaneous photon collection from the two modes and further detection with APDs allow us to obtain a sequence of pulses ( $p$-time trace) from which PDFs and $g_{p}^{(2)}$ values are computed. Bottom: "Singlephoton detection" scheme. Light from the bonding mode is also sent to a HBT setup to provide direct measurement of second-order coherence, used as a cross-check. SNSPD stands for superconducting nanowire single-photon detector; FBS stands for fibered beam splitter. Inset: Normalized coincidence count histogram of the B-mode for $P=4 P_{\text {th }}$. (b) Circles: $g_{p}^{(2)}$ for $B$ and $A B$ modes at different pump powers computed from the variance of the $p$-time traces shown in (a). Black diamonds : area under the central peak of the HBT autocorrelation normalized to the average area under secondary peaks (error bars in grey shadow), in good agreement with $g_{p}^{(2)}$ measurements (see Supplemental Material [25], Sec. F, for a theoretical comparison).

eventually calibrated in photon number $(p)$ : we call this method single-pulse energy detection (SPED). Typical $p$-time series contain $10^{4}$ pulses. Figure 2(a) shows a segment of two simultaneous $p$-time series displaying both the AB-mode and B-mode signals. We point out that, since the cavities operate in a laser regime, each output pulse, containing projections on both $\mathrm{B}$ and $\mathrm{AB}$ modes, results 
from a single trajectory in phase space, whose time integration leads to a single-pulse photon number.

Second-order correlations of the pulse energies $g_{u}^{(2)}$ (see Supplemental Material [25], Sec. F) or equivalently, photon number $g_{p}^{(2)}$, are computed from the variances of the $p$-time traces and depicted in Fig. 2(b) for both modes. Our method is validated by means of the standard Hanbury Brown and Twiss (HBT) experiments [see Fig. 2(a) lower frame and Appendix B] $[29,30]$. Note that these correlation traces are normalized time-integrated autocorrelation functions $\tilde{R}(\tau)$ (see Appendix B), whose central peak can be approximated as snapshots of $g^{(2)}(t, \tau)$ close to the pulse maximum, $\tilde{R}(0) \approx g^{(2)}\left(t_{\max }, 0\right) \quad$ (see Supplemental Material [25], Sec. F). It is clearly observed in Fig. 2(b) that $g_{p}^{(2)}$ with the SPED method and the normalized area under the central correlation peak with the HBT method are within the experimental errors. In addition, $g_{p}^{(2)}$ for the B mode reveals superthermal emission $\left[g_{p}^{(2)}>2\right]$ for $P \gtrsim 1.5 P_{\mathrm{th}}$, while the AB-mode emission remains Poissonian $\left[g_{p}^{(2)} \approx 1\right]$.

Figures 3(a)-3(c) show experimental and numerical photon number PDFs for three intercavity coupling parameters obtained using the barrier engineering technique [31], $K=3.3, K=6$, and $K=10$, respectively, and three different pump rates, $P=1.5 P_{\mathrm{th}}, P=2 P_{\mathrm{th}}$, and $P=$ $3 P_{\text {th }}$ from left to right. For the two larger coupling parameters $(K=6$ and $K=10)$, the PDFs evolve from nearly exponential to long-tailed PDFs as the pump power is increased, showing very good agreement between experimental data and numerical simulations of a stochastic mean-field model. As for second-order moments, the measured PDF can also be interpreted as snapshots close to pulse maximum $t=t_{\max }$ : a stronger pump induces more intense laser output pulses, and hence a stronger quench, which leads to more pronounced long-tailed distributions.

Now, a link can be established between the timedependent evolutions of the statistical observables after quench and the experimental results obtained with the single-pulse energy detection scheme. We note that the two larger coupling parameters, $K=6$ and $K=10$, belong to the quasilinear, large-coupling Rabi regime for which $K>\alpha$ [32] (here $\alpha=5$ ). The superthermal quenches asymptotically reach $g_{p}^{(2)}$ values of approximately 3-3.5 [Figs. 3(e)-3(f), left], in agreement with $g^{(2)}\left(t_{\max }, 0\right) \approx 3$ in Fig. 1(a). The deviation from equilibrium can be quantified by computing the excess entropy $\Delta S_{i}$ as defined in Eq. (10) but using pulse energies [Supplemental Material [25], Sec. J, Eq. (S72)]. The monotonic entropy increase toward $\Delta S_{i} \sim 0.2-0.3$ is compatible with transients after quench for $K=6$ and $K=10$ and corresponds to $\Delta S_{i}\left(t_{\max }\right)$ predicted by the model [see Fig. 1(a)].

Unlike large $K$-values, the case $K=3.3$ is fundamentally different as it jumps into the Josephson regime, $K<\alpha$ [32]. Here, neither $g_{p}^{(2)}$ nor the excess entropy have the same behavior as for very strong coupling. In addition, it can be observed that $g_{p}^{(2)}$ tends to 1 for increased pumping, which is an indication of the presence of a coherent phase. The local maxima in the distributions for $K=3.3$ [Fig. 3(a)] lead to larger deviations from equilibrium which, even in good agreement with the full numerical simulations of the stochastic mean-field model, are not predicted by the simple 1D model [Eq. (6)]. Instead, the large entropy departure for $K=3.3$ and $P>2.5 P_{\text {th }}$ [Fig. 3(d), right] is observed, which can be interpreted as a consequence of symmetry-breaking
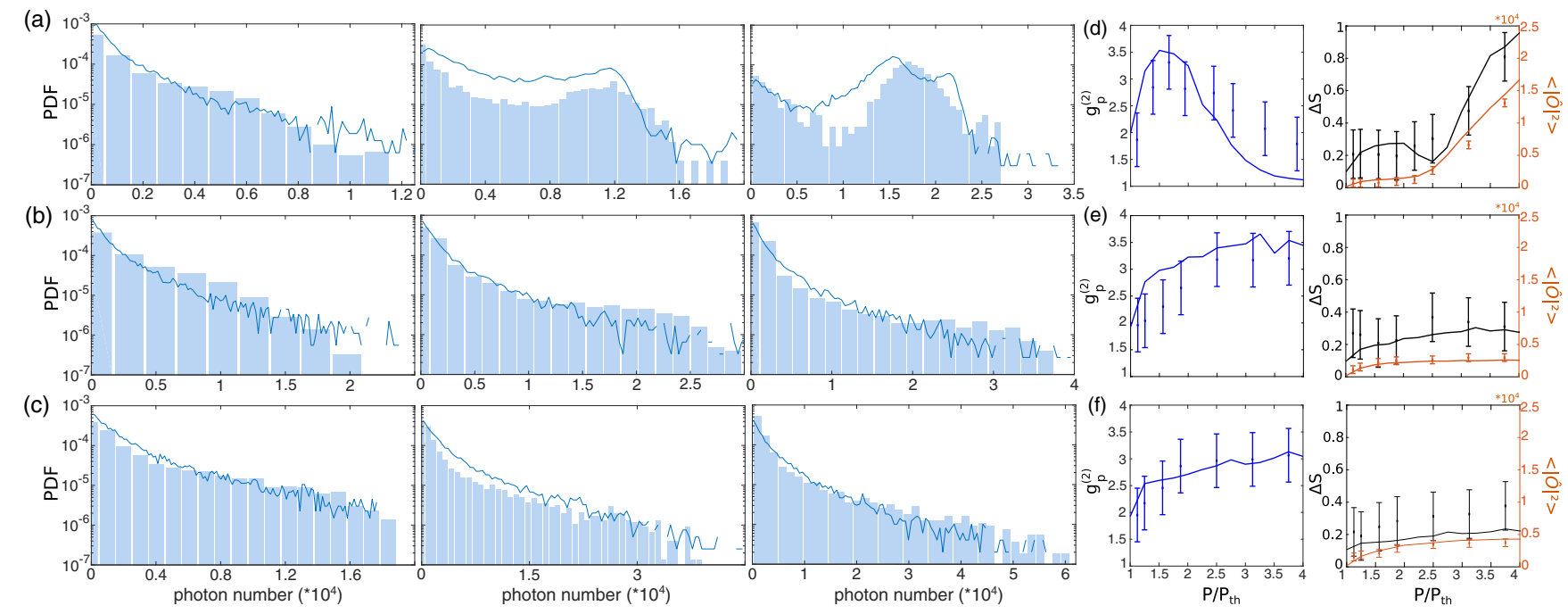

FIG. 3. Statistical distributions of the photon number for the $B$-mode. (a)-(c) Experimental (bars) and theoretical (lines) PDFs for increased pump powers $P=1.5 P_{\text {th }}$ (left), $P=2 P_{\text {th }}$ (middle), and $P=3 P_{\text {th }}$ (right) and different intercavity coupling levels: (a) $K=3.3$, (b) $K=6$, and (c) $K=10$. (d)-(f) Experimental (symbols) and theoretical (lines) degree of second-order coherence (left); excess entropy (right, black); and order parameter (right, orange) as a function of $P$ for (d) $K=3.3$, (e) $K=6$, and (f) $K=10$. In the numerical simulations, $\gamma=-0.1, \alpha=5$, and $\gamma_{\text {tot }}^{-1}=28$. 
phenomena taking place for such coupling parameters, as we have recently explored in continuous wave operation [19]. As proposed in Ref. [21], a symmetry-breaking phase transition can be detected from the evolution of an order parameter, $|\hat{O}|=\left|A_{1}+A_{2}\right|$, which will grow up under symmetry-breaking conditions. We observe in Figs. 3(d)3(f) (right panels) that $\left\langle|\hat{O}|^{2}\right\rangle$ remains small for $K \geq 6$, while it experiences an abrupt increase for $K=3.3$ and $P>2.5 P_{\text {th }}$, which constitutes experimental evidence-in the sense of a quench - of a symmetry-breaking phase transition. Hence, $K=3.3$ with $P>2.5 P_{\text {th }}$ corresponds to the mixing of a thermal state with a symmetry-broken coherent phase, which is beyond the simple long-tailed distributions described in Sec. II. It shows the increasing statistical complexity of the dissipative coupled cavity system when accessing the highly nonlinear Josephson limit in which the nonlinear laser frequency shift overcomes the tunnel energy $K$ [19].

\section{ANALOGY WITH BROWNIAN PARTICLES}

This section aims at addressing the universality of our farfrom-equilibrium distributions after quench, by mapping the nanolaser system to the dynamics of Brownian particles in a fluid. Brownian particles are mesoscopic objects subjected to external forces but also in contact with a thermal bath, e.g., pollen submerged into a fluid as in the original Brown experiment [33]. The interactions with the thermal reservoir provide at the same time dissipation and fluctuations that lead to thermal equilibrium. In the high friction limit, the altitude $x_{j}$ of the particle $j$ follows the Langevin equation of motion $\gamma d x_{j} / d t=-g+\sqrt{2 \gamma k_{B} T / m} \xi_{j}(t)$, where $\gamma$ is the viscous damping rate, $g$ is gravitational acceleration, $k_{B}$ the Boltzmann constant, $m$ the mass, $T$ the reservoir temperature, and $\xi_{j}(t)$ a Gaussian white noise. We consider a hard (reflecting) wall at $x=0$ as the boundary condition and rescale the variables as $T \rightarrow T / \theta_{g}$ with the characteristic temperature $\theta_{g}=g^{2} m / \gamma^{2} k_{B}, x \rightarrow x \gamma^{2} / g$, and $t \rightarrow \gamma t$. The normalized Langevin equation has the form of Eq. (6), but now $f(x)=-1$ and $g^{2}=2 T$. The equilibrium solution of the FP equation [Eq. (7)] is also given by Eq. (8), i.e., an exponential distribution which, in this simple case, is that of the potential energy $U(x)=x$ [see Figs. 4(a) and 4(c)], with $\beta=1 / T$ and $\mathcal{N}=\beta$.

We point out that the transient dynamics toward equilibrium is governed by the viscosity $\gamma$. As a consequence, if the system is initially at thermal equilibrium with temperature $T=T_{1}$, two widely different paths, or cooling processes, are possible toward a temperature $T_{2}<T_{1}$. First, the reservoir temperature $T(t)$ may decrease slowly as compared to the characteristic time scale of the particle $\gamma^{-1}$. In our rescaled units, this means $\dot{T} / T \ll 1$. In this adiabatic case, the distribution during the cooling process is defined by the equilibrium distribution with a time-dependent temperature $\rho(x, t)=\rho_{\text {eq }}[x ; T(t)]$ (see Supplemental Material [25], video $\mathrm{S} 1)$.
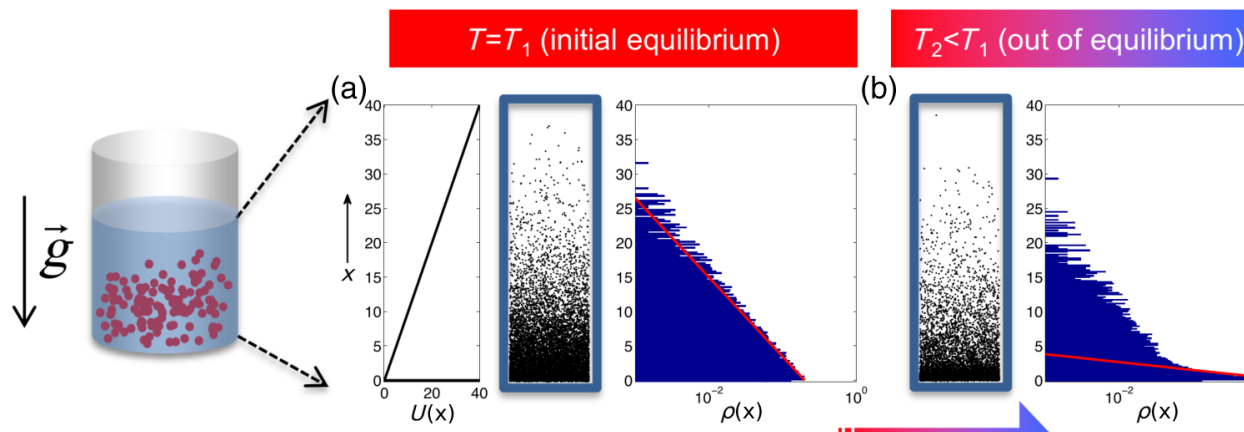

(b)

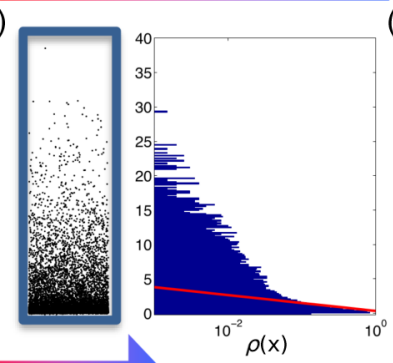

quenching

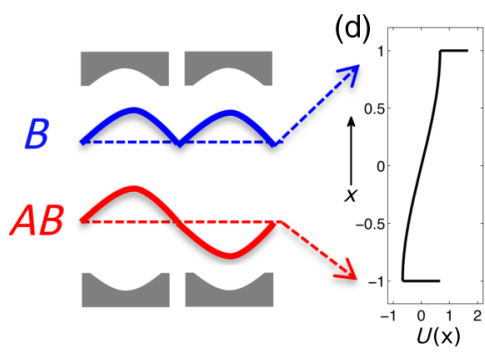

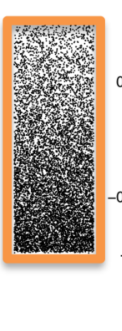

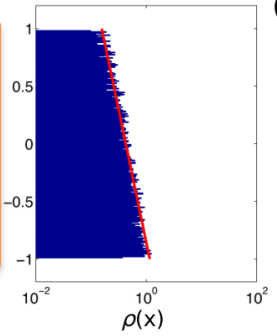

(e)

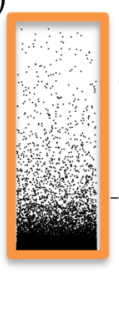

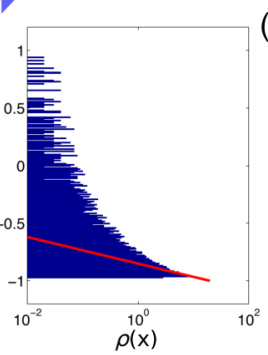

$T=T_{2}$ (final equilibrium)

(c)

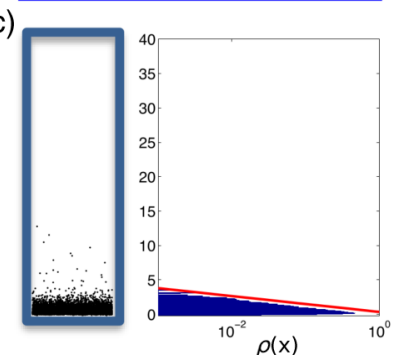

(f)

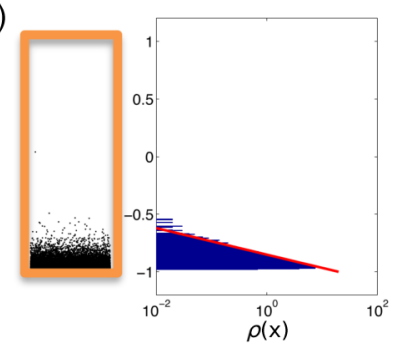

FIG. 4. Quenching analogy between a suspension of Brownian particles in a fluid (a)-(c) and a double cavity laser with mode energy flow from bonding $(B)$ to antibonding $(A B)$ modes (d)-(f). (a), (d): Initial equilibrium; from left to right: potential energy, a snapshot of the physical ensemble ( $10^{4}$ particles), height histogram (blue bars), and the steady-state equilibrium distribution $\rho_{\text {eq }}(x, T)$ [red line, Eq. (8)]. (b), (e): Quenched phase. The far nonequilibrium distributions are due to the abrupt decrease of the reservoir temperature from $T_{1}$ to $T_{2}<T_{1}$. Particles located at high altitudes remain "hot," while lower particles cool down more rapidly. (c)-(f) Final equilibrium at $T=T_{2}$. 
Another possibility consists in decreasing the temperature abruptly. The system will remain in an out-of-equilibrium state for some time, with a statistical distribution that will not correspond to the exponential equilibrium [see Fig. 4(b) and Supplemental Material [25], video S2). In such a transient phase after quench, the particles are simply falling and the Brownian fluctuations can be neglected; hence, the dynamics is dominated by transport (drift) rather than diffusion. Collisions with the bottom wall make particles thermalize faster: those with low potential energy become cold, whereas high-altitude particles remain hot. This simple picture unveils quench phenomena in more complex systems such as our strongly coupled cavities operating in the laser regime [Figs. 4(d)-4(f)].

The analogy between falling Brownian particles and compound cavity laser systems relies on three important features: (i) coupled cavities may support several (here two) eigenmodes which, as long as the cavities are filled with active (gain) media, interact through mode-mode scattering and/or gain competition leading to energy flow (drift) among the modes [32,34]; (ii) stochastic fluctuations are intrinsic because of spontaneous emission noise; and (iii) the equilibrium below laser threshold is thermal. Within this analogy, the reservoir temperature in the coupled cavity system is given by $\beta^{-1} \sim S_{\mathrm{sp}} I^{-1}$. Hence, a rapid intensity increase in the bimodal laser corresponds to a fast cooling of the Brownian particle system. A main difference can still be pointed out: the stochastic process in the Brownian particle case is given by additive noise, while the noise term is multiplicative in the optical system. Also, the potential is nonlinear in the optical system while it is linear in the falling Brownian particle case. Remarkably, the drift and diffusion terms combine together in the optical system in such a way that the equilibrium is also exponential. Physically, the drift force in the bimodal nanolaser results from loss difference between the two optical modes.

\section{CONCLUSIONS}

In conclusion, we have shown a far-from-equilibrium route to superthermal emission in coupled nanolasers by preparing the system with a short-pump-pulse parameter quench. The signatures of nonequilibrium transients are heavy-tailed photon distributions of the weak hybrid mode, which have been experimentally observed by means of a single-pulse energy detection scheme. The universality of this process relies on an abrupt effective temperature decrease in a 1D stochastic transport equation for the mode energy: the drift forces overcome diffusion, leading to super-exponential distributions with "high temperature" deviations. The distance from equilibrium has been quantified through the excess internal entropy, which could be used as a nonarbitrary indicator of heavy-tail statistics in, e.g., the physics of extreme events [35]. Finally, our farfrom-equilibrium mechanism is illustrated through the analogy with an ensemble of a suspension of Brownian particles in a fluid upon a fast-cooling process. These results open up a new paradigm to investigate classical phase transitions and out-of-equilibrium thermodynamics using multimode nonlinear dissipative optical cavities.

\section{ACKNOWLEDGMENTS}

We acknowledge Nadia Belabas for helping with the use of superconducting nanowire single photon detectors, and Isabelle Robert-Philip, Sébastien Tanzilli and Olivier Alibart for discussions on HBT experiment. This work is supported by the "Investissements d'Avenir" program (Labex NanoSaclay, Grant No. ANR-10-LABX-0035), the ANR UNIQ DS078, and the RENATECH network. J. J. acknowledges the financial support from the project COMBINA (TEC2015-65212-C3-3-P MINECO/FEDER UE) and the Ramón y Cajal fellowship.

\section{APPENDIX A: PHOTONIC CRYSTAL NANOLASER FABRICATION}

The two coupled nanocavities are made as defects (three holes missing) in a photonic crystal lattice consisting of air holes etched in a 265-nm-thick InP membrane. The InP membrane contains four InGaAs/InGaAsP quantum wells with photoluminescence centered at $1510 \mathrm{~nm}$ and $\mathrm{FWHM}=63 \mathrm{~nm}$. It is bonded to a silicon substrate by means of a 400-nm benzocyclobutene (BCB) layer. In between $\mathrm{BCB}$ and $\mathrm{InP}$, a $1-\mu \mathrm{m}$-thick $\mathrm{SiO}_{2}$ layer is chemically underetched in order to suspend the InP membrane and provide a Q-factor of about 5000. The amount of evanescent coupling between the nanolasers can be precisely tailored by modifying the hole diameter of the central row in the barrier [31].

\section{APPENDIX B: HANBURY BROWN AND TWISS EXPERIMENT}

The bonding mode intensity is split into two identical paths with a 50/50 fibered beam splitter. Each output is sent to superconducting nanowire single-photon detectors (SNSPD, Single Quantum Eos, closed-cycle systems). The quantum efficiency of these devices is about $15 \%$ and the timing jitter is $50 \mathrm{ps}$. We operate the SNSPD with a bias current well below the superconductor threshold so that we only count 50 dark photons per second. To perform coincidence measurements, we attenuate the signal down to 2000 counts/s on each detector. SNSPD outputs are monitored with an oscilloscope (Lecroy Wavepro $725 \mathrm{Zi}$ $2.5 \mathrm{GHz}, 40 \mathrm{GS} / \mathrm{s})$. The coincidence count diagram is obtained by triggering the oscilloscope on one SNSPD channel, and we use the built-in Lecroy function « skew 》 to measure the time interval from the closest detection event on the second SNSPD channel. Then, a histogram of the time intervals is plotted on the oscilloscope with 1000 
detection events. With this method, we thus reconstruct the time-integrated autocorrelation function $R(\tau)$. We define $\tilde{R}(\tau)=\left\{R(\tau) /\left[[1 /(2 M)] \overline{\sum_{-M(m \neq 0)}^{M} R(\tau=m T)}\right]\right\} \quad$ as the autocorrelation function normalized to the side-peaks average value. Therefore, the degree of second-order coherence in the energy variable is the area under the central peak of the normalized autocorrelation function divided by the average of the areas under the side peaks, $g_{u}^{(2)} \approx\left\{\left[\int_{-T / 2}^{T / 2} \tilde{R}(\tau) d \tau\right] /\right.$ $\left.\int_{\text {side-peaks }} \tilde{R}(\tau) d \tau\right\}$ (see Supplemental Material [25], Sec. F).

[1] A. Jechow, M. Seefeldt, H. Kurzke, A. Heuer, and R. Menzel, Enhanced Two-Photon Excited Fluorescence from Imaging Agents Using True Thermal Light, Nat. Photonics 7, 973 (2013).

[2] P. Ryczkowski, M. Barbier, A. Friberg, J. M. Dudley, and G. Genty, Ghost Imaging in Time Domain, Nat. Photonics 10, 167 (2016).

[3] J. Wiersig, C. Gies, F. Jahnke, M. Assmann, T. Berstermann, M. Bayer, C. Kistner, S. Reitzenstein, C. Schneider, S. Höfling, A. Forchel, C. Kruse, J. Kalden, and D. Hommel, Direct Observation of Correlations between Individual Photon Emission Events of a Microcavity Laser, Nature (London) 460, 245 (2009).

[4] Y. Zhou, F.-1. Li, B. Bai, H. Chen, J. Liu, Z. Xu, and H. Zheng, Superbunching Pseudothermal Light, Phys. Rev. A 95, 053809 (2017).

[5] F. Jahnke, C. Gies, M. Aßmann, M. Bayer, H. A. M. Leymann, A. Foerster, J. Wiersig, C. Schneider, M. Kamp, and S. Höfling, Giant Photon Bunching, Superradiant Pulse Emission and Excitation Trapping in Quantum-Dot Nanolasers, Nat. Commun. 7, 11540 (2016).

[6] H. A. M. Leymann et al., Intensity Fluctuations in Bimodal Micropillar Lasers Enhanced by Quantum-Dot Gain Competition, Phys. Rev. A 87, 053819 (2013).

[7] C. Redlich et al., Mode-Switching Induced Super-Thermal Bunching in Quantum-Dot Microlasers, New J. Phys. 18, 063011 (2016).

[8] H. E. Kondakci, A. Szameit, A. F. Abouraddy, D. N. Christodoulides, and B.E.A. Saleh, Sub-Thermal to Super-Thermal Light Statistics from a Disordered Lattice via Deterministic Control of Excitation Symmetry, Optica 3, 477 (2016).

[9] H. Kondakci, A. F. Abouraddy, and B. E. A. Saleh, A Photonic Thermalization Gap in Disordered Lattices, Nat. Phys. 11, 930 (2015).

[10] M. Cheneau, P. Barmettler, D. Poletti, M. Endres, P. Schausz, T. Fukuhara, C. Gross, I. Bloch, C. Kollath, and S. Kuhr, Light-Cone-Like Spreading of Correlations in a Quantum Many-Body System, Nature (London) 481, 484 (2012).

[11] A. Tomadin, V. Giovannetti, R. Fazio, D. Gerace, I. Carusotto, H. E. Türeci, and A. Imamoglu, Signatures of the Superfluid-Insulator Phase Transition in Laser-Driven Dissipative Nonlinear Cavity Arrays, Phys. Rev. A 81, 061801(R) (2010).
[12] C. Creatore, R. Fazio, J. Keeling, and H. E. Türeci, Quench Dynamics of a Disordered Array of Dissipative Coupled Cavities, Proc. R. Soc. A 470, 20140328 (2014).

[13] S. Raghavan, A. Smerzi, S. Fantoni, and S. R. Shenoy, Coherent Oscillations between Two Weakly Coupled BoseEinstein Condensates: Josephson Effects, $\pi$ Oscillations, and Macroscopic Quantum Self-Trapping, Phys. Rev. A 59, 620 (1999).

[14] T. Zibold, E. Nicklas, C. Gross, and M. K. Oberthaler, Classical Bifurcation at the Transition from Rabi to Josephson Dynamics, Phys. Rev. Lett. 105, 204101 (2010).

[15] A. S. Rodrigues et al., in Spontaneous Symmetry Breaking, Self-Trapping, and Josephson Oscillations (Springer, Berlin, 2013), pp. 509-529.

[16] M. Abbarchi, A. Amo, V. G. Sala, D. D. Solnyshkov, H. Flayac, L. Ferrier, I. Sagnes, E. Galopin, A. Lemaitre, G. Malpuech, and J. Bloch, Macroscopic Quantum SelfTrapping and Josephson Oscillations of Exciton Polaritons, Nat. Phys. 9, 275 (2013).

[17] M. Albiez, R. Gati, J. Fölling, S. Hunsmann, M. Cristiani, and M. K. Oberthaler, Direct Observation of Tunneling and Nonlinear Self-Trapping in a Single Bosonic Josephson Junction, Phys. Rev. Lett. 95, 010402 (2005).

[18] S. R. K. Rodriguez, A. Amo, I. Sagnes, L. Le Gratiet, E. Galopin, A. Lemaitre, and J. Bloch, Interaction-Induced Hopping Phase in Driven-Dissipative Coupled Photonic Microcavities, Nat. Commun. 7, 11887 (2016).

[19] P. Hamel, S. Haddadi, F. Raineri, P. Monnier, G. Beaudoin, I. Sagnes, A. Levenson, and A. Yacomotti, Spontaneous Mirror-Symmetry Breaking in Coupled Photonic-Crystal Nanolasers, Nat. Photonics 9, 311 (2015).

[20] A. Trenkwalder, G. Spagnolli, G. Semeghini, S. Coop, M. Landini, P. Castilho, L. Pezze, G. Modugno, M. Inguscio, A. Smerzi, and M. Fattori, Quantum Phase Transitions with Parity-Symmetry Breaking and Hysteresis, Nat. Phys. 12, 826 (2016).

[21] W. Casteels and C. Ciuti, Quantum Entanglement in the Spatial-Symmetry-Breaking Phase Transition of a Driven-Dissipative Bose-Hubbard Dimer, Phys. Rev. A 95, 013812 (2017).

[22] B. Cao, K. W. Mahmud, and M. Hafezi, Two Coupled Nonlinear Cavities in a Driven-Dissipative Environment, Phys. Rev. A 94, 063805 (2016).

[23] J. Marino and S. Diehl, Driven Markovian Quantum Criticality, Phys. Rev. Lett. 116, 070407 (2016).

[24] J. Raftery, D. Sadri, S. Schmidt, H. E. Türeci, and A. A. Houck, Observation of a Dissipation-Induced Classical to Quantum Transition, Phys. Rev. X 4, 031043 (2014).

[25] See Supplemental Material at http://link.aps.org/ supplemental/10.1103/PhysRevX.8.011013 for additional theoretical and experimental analysis and figures.

[26] P. Rice and F. J. Carmichael, Photon Statistics of a Cavity-QED Laser: A Comment on the Laser Phase Transition Analogy, Phys. Rev. A 50, 4318 (1994).

[27] J. P. Woerdman, M. P. Van Exter, and N. J. Van Druten, Quantum Noise of Small Lasers, Adv. At. Mol. Opt. Phys. 47, 205 (2001).

[28] S. M. Ulrich, C. Gies, S. Ates, J. Wiersig, S. Reitzenstein, C. Hofmann, A. Löffler, A. Forchel, F. Jahnke, and 
P. Michler, Photon Statistics of Semiconductor Microcavity Lasers, Phys. Rev. Lett. 98, 043906 (2007).

[29] R. Hanbury Brown and R. Q. Twiss, A Test of a New Type of Stellar Interferometer on Sirius, Nature (London) 178, 1046 (1956).

[30] Y. Bromberg, Y. Lahini, E. Small, and Y. Silberberg, Hanbury Brown and Twiss Interferometry with Interacting Photons, Nat. Photonics 4, 721 (2010).

[31] S. Haddadi, P. Hamel, G. Beaudoin, I. Sagnes, C. Sauvan, P. Lalanne, J. A. Levenson, and A. M. Yacomotti, Photonic Molecules: Tailoring the Coupling Strength and Sign, Opt. Express 22, 12359 (2014).
[32] M. Marconi, J. Javaloyes, F. Raineri, A. Levenson, and A. Yacomotti, Asymmetric Mode Scattering in Strongly Coupled Photonic Crystal Nanolasers, Opt. Lett. 41, 5628 (2016).

[33] P. Hänggi and F. Marchesoni, Introduction: 100 Years of Brownian Motion, Chaos 15, 026101 (2005).

[34] H. A. M. Leymann et al., Pump-Power-Driven Mode Switching in a Microcavity Device and Its Relation to Bose-Einstein Condensation, Phys. Rev. X 7, 021045 (2017).

[35] M. Onorato, S. Residori, U. Bortolozzo, A. Montina, and F. T. Arecchi, Rogue Waves and Their Generating Mechanisms in Different Physical Contexts, Phys. Rep. 528, 47 (2013). 